\title{
Numerical simulations of self-oscillatory flows near blunted bodies, giving off opposite jets
}

\author{
Bladimir Ivanovich Pinchukov \\ Siberian division of Russian Academy of Sc., In-te of Computational Technologies, Novosibirsk, 630090, Russia
}

Email address:

pinchvi@ict.nsc.ru

\section{To cite this article:}

Bladimir Ivanovich Pinchukov. Numerical Simulations of Self-Oscillatory Flows near Blunted Bodies, Giving off Opposite Jets. International Journal of Mechanical Engineering and Applications. Vol. 2, No. 1, 2014, pp. 5-10. doi: $10.11648 /$ j.ijmea.20140201.12

\begin{abstract}
New self-oscillatory compressible flows are found and investigated. Self-oscillations are supposed to be produced as a result of resonance interactions of flow "active" elements, namely, elements, amplifying disturbances. Hypothesis is used that contact discontinuities and intersection points of shocks with shocks or shocks with contact discontinuities compose the flow set of "active" elements. Two-dimensional Reynolds-averaged Navier-Stocks equations added by an algebraic turbulence model are solved by an implicit third order Runge-Kutta scheme. Well studied open cavity flow and jet impinging on a plane are calculated to verify the numerical method and the turbulence model. Compressible flows near blunted bodies, giving off supersonic opposite jets from forehead surfaces, are discovered to have self-oscillatory regimes.
\end{abstract}

Keywords: Self-Oscillatory Flows, Reynolds-Averaged Navier-Stocks Equations, High Resolution Methods, Runge-Kutta Schemes

\section{Introduction}

A jet cavity interactions (see, for example, [1-5]) are known to have self-oscillatory regimes. Papers [1-4] are devoted to investigations of various aspects of main frequencies dependences on geometrical parameters of jets and environments. Kastner and Samimy [5] studied a Hartmann cavity in which the region between the jet and the cavity is shielded partly.

A jet impinging on a plane [6-14] is another type of compressible jet unsteady flows. Self-oscillations main frequencies are studied in [6-7]. Numerical flow field simulations are carried out in [8-14]. The numerical approach LES is used in [10-12]. RANS, added by an algebraic turbulence model, are used in [13-14]. An impinging of a supersonic underexpanded jet, surrounded by a subsonic stream, on a blunted cone, was found also to have self-oscillatory regimes [15].

Main purpose of the recent paper is to extend this list of jet unsteady flows by means of a numerical search. We construct and investigate flows, containing "active" elements. Particularly, flows near blunted bodies (cylinders or cones), giving off opposite jets, were discovered to have unsteady regimes $[14,16]$. Here these investigations are continued.
The mentioned above mechanism of self-oscillations is based on resonance interactions of flow "active" elements, namely, elements, which amplify disturbances. We suppose that contact discontinuities and intersection points of shocks with shocks or shocks with contact discontinuities compose the flow set of "active" elements. Possibility of the disturbances amplification by contact discontinuities is a result of the Kelvin-Helmholtz instability and is accepted. Inclusion of intersection points to a list of amplifiers [13-14] is initiated by the known possibility to operate types of the shock reflection from a plane (Mach or regular type) by small influences. If small influences yield significant change of the flow in the reflection zone, then this flow structure is an amplifier of disturbances. Possibility of any intersection points of discontinuities (intersection lines in $3 \mathrm{~d}$ case) to amplify disturbances is used here as a hypothesis, which is checked by results of a search for new unsteady flows.

Since active flow elements, mentioned above, amplify disturbances, that yields possibility of interactions between any these elements. Namely, disturbances from one element affect at the flow near some another. Change of the flow near the last element affect at the flow near initial one. If reflected 
disturbances come back to the initial element with nearly the same phase, as this element emits at that moment, then conditions are formed for the positive feedback effect. As a result, a resonance may take place, which induces flow self-oscillations.

The body surface reflects disturbances and, consequently, also plays a rule in producing of self-oscillations. Naturally, this effect depends on the surface form. For example, numerical investigations [15] of interactions blunted cones with jets, surrounded by subsonic streams, show significant decreasing of the self-oscillations amplitude when the plain cone blunt is replaced by the spherical blunt, which disperses reflected disturbances. Unlike "active" elements, body surfaces do not increase disturbances energy and plays a secondary rule in self-oscillations producing. Availability of "active" elements does not guarantee producing of self-oscillations and may provide also an existence of steady regimes.

There is possibility of three types of interaction:

1 - a contact discontinuity with a contact discontinuity;

2 - an intersection point with a contact discontinuity;

$3-$ an intersection point with an intersection point.

\section{Physical and Computational Models}

Reynolds-averaged Navier-Stocks equations added by an algebraic turbulence model are used here and in previous author's investigations [13-16]. A search for new self-oscillatory flows deals with trial calculations of numerous different flows. So, we need in a simple and universal turbulence model. Here the model is used, based on the Prandtl formulae

$$
\mu=\rho|w| k^{2} z^{2}
$$

where $w$ is a vorticity, $\rho$ is density, $z$ is the length scale, $k=0.4$ is the von-Karman constant. This formulae is dealt in classical Cebeci-Smith and Baldween-Lomax models, where the length scale is defined as the recent point distance to the solid wall. This formulae is used also in the Smagorinsky model of LES (Large Eddy Simulation), where the length scale is the grid cell size. Another definition is used here. We divide flows on vortex zones and ideal zones. The length scale is equal to zero in ideal zones and is equal to the recent point distance to the zone boundary in vortex zones, with next usage of the limitation written below.

The viscosity calculation starts with the determination of vortex zones by the velocity circulation calculation. The current mesh cell belongs to any vortex zone if the inequality is true:

$$
\begin{aligned}
& \left|w_{i+1 / 2, k+1 / 2}\right| S_{i+1 / 2, k+1 / 2}=\mid\left(u_{i+1 / 2, k} \Delta r_{i+1 / 2, k}\right)- \\
& -\left(u_{i+1 / 2, k+1} \Delta r_{i+1 / 2, k+1}\right)+\left(u_{i+1, k+1 / 2} \Delta r_{i+1, k+1 / 2}\right)- \\
& -\left(u_{i, k+1 / 2} \Delta r_{i, k+1 / 2}\right) \mid>e\left\{\left|u_{i+1 / 2, k}\right|\left|\Delta r_{i+1 / 2, k}\right|+\right. \\
& +\left|u_{i+1 / 2, k+1}\right|\left|\Delta r_{i+1 / 2, k+1}\right|+\left|u_{i+1, k+1 / 2}\right|\left|\Delta r_{i+1, k+1 / 2}\right|+ \\
& \left.+\left|u_{i, k+1 / 2}\right|\left|\Delta \boldsymbol{r}_{i, k+1 / 2}\right|\right\},
\end{aligned}
$$

where $S$ is the mesh cell area, $\boldsymbol{u}$ are velocity vectors at mesh nodes, $\Delta \boldsymbol{r}$ are vectors connecting neighbouring nodes, $e$ is a small constant (for which the value $3 / N$ is chosen in trial calculations, $N$ - the most number of mesh points in space variables), $(u \Delta r)$ are scalar multiplications of vectors, $|u||\Delta r|$ are multiplications of vector lengths. The middle part of this relation represents approximately the velocity circulation along mesh cell boundaries. The length scale $z$ is calculated by formulas:

$$
\mathrm{z}=\mathrm{d}\left[\mathrm{x}-(\mathrm{x} / 1.5)^{3} / 2\right], \text { if } \mathrm{x}=\mathrm{L} / \mathrm{d}<1.5, \mathrm{z}=\mathrm{d}, \text { if } \mathrm{x}>1.5 .
$$

where $L$ - the recent point distance to the vortex zone boundary, $d-$ a delimiting parameter. These formulas define a delimiter with continuous first derivative. Calculations, presented here, are carried out for $d=r / 60, r$ - the radius of spherical blunt of considered bodies or the jet radius in investigations of a supersonic jet impinging on a plane. Trial calculations with usage the constant length scale $z=d$ show worse resolution of small flow details compared with the written above approach (numerical illustrations are presented in fourth chapter).

The used here approach deals with the fixed aggregate of scales of disturbances represented in the turbulent viscosity. As a result, the turbulent viscosity is steady while the mesh number is varying and, consequently, the contact discontinuities thickness (more precisely, shift layers in our case) is steady while the mesh number is varying. It allows simple checking of the solutions convergence in the mesh nodes number and, consequently, allows to get additional verification of the unsteady regimes existence. Since we search for new self-oscillatory flows, which are not studied experimentally, this verification is a particularly important component of our investigations.

An implicit conservative Runge-Kutta scheme [15,17] is employed here, which is third order in time and fourth order in space (viscous terms are approximated with second order) This scheme version, which has the same space stencil but which is first order in time is used for additional verification of the self-oscillatory regimes existence.

Naturally, numerical calculations deal with dimensionless variables. These variables are defined as relations of initial variables and next parameters of the undisturbed flow or the body size: $p_{\infty}$ - for pressure, $\rho_{\infty}$ - for density, $\sqrt{p_{\infty} / \rho_{\infty}}$ for velocity, $r$ (blunt radiuses of cones or cylinders) - for space variables, $r / \sqrt{p_{\infty} / \rho_{\infty}}$ - for time.

\section{Test Problems}

An open cavity flow [18-19] and an underexpanded supersonic jet impinging on a plane [20-21] are chosen for comparison to verify the numerical method and the turbulence model.

The cavity depth (see figure 1 ) is $5.2 \mathrm{~mm}$, the cavity length is $10.4 \mathrm{~mm}$, the numerical region above cavity has the height of $10.4 \mathrm{~mm}$ and the length of $20.8 \mathrm{~mm}$. This cavity flow is 
tested numerically with flow field conditions $M_{\infty}=2$, $\theta=0.979 \mathrm{~mm}$ (momentum thickness of the boundary layer on the inflow plane). Boundary conditions for computations are no-slip adiabatic wall on solid surfaces, extrapolations on the outflow boundaries, prescribed variables on the inflow plane. Namely, dimensionless pressure and density are 1 , the vertical velocity component $v$ is 0 , the horizontal velocity component is $u=M_{\infty} \sqrt{\gamma}(2 \sqrt{s}-s)$ if $s=y / \theta \leq 10$, $u=M_{\infty} \sqrt{\gamma}$ if $s=y / \theta>10, \gamma=1.4-$ the specific heat ratio.

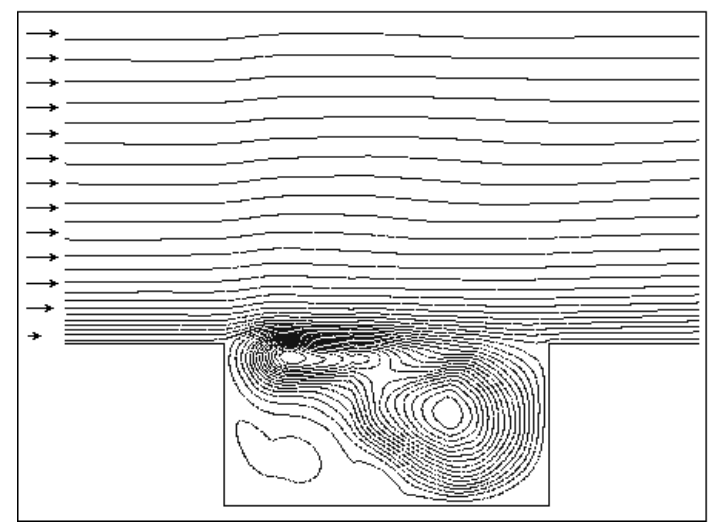

Figure 1a. Cavity flow streamlines

The time history of the surface pressure at the $x=2 L / 3$ point on the cavity floor is used to form the time averaged sound pressure level $\overline{S P L}$, which is computed by the equation

$$
\overline{S P L}=10 \log _{10}\left(\overline{p^{\prime 2}} / p_{r e f}^{2}\right)
$$

where

$$
\overline{p^{\prime 2}}=\sum_{n}\left(\mathrm{p}_{n}-\bar{p}\right)^{2} / \mathrm{N}, \mathrm{p}_{r e f}=20 \mathrm{mkPa} / \mathrm{p}_{\infty}
$$

$p_{\infty}=98066 \mathrm{~Pa}$ (air pressure under normal conditions) is used since dimensionless variables are dealt here. The resulting time averaged $\overline{S P L}$ of $174.8 \mathrm{Db}$ may be compared with the numerical $\overline{S P L}$ of $167.54 \mathrm{Db}$ and the experimental $\overline{S P L}$ of $164.41 \mathrm{Db}$ presented in [18]. The weighted $\overline{S P L}$ for data from various sources [19] is approximately $171 \mathrm{Db}$.

An underexpanded supersonic jet impinging on a plane [20-21] may illustrate the proposed self-oscillations mechanism. Figure 2 shows the density distribution between two infinite planes. The jet outflows from the axisymmetric nozzle on the left plane in figure 2. The supposition is used that the temperature at a centre of the nozzle exit cross section is equal to the surrounding air temperature. Jet parameters are computed by the system of algebraic equations, which describes the one-dimensional flow from a point source. The horizontal velocity component is equal to zero on solid surfaces (vertical boundaries in figure 2), the radial velocity component, pressure and density are extrapolated. The radial velocity component is equal to zero on the symmetry axis, other variables are extrapolated. Extrapolation conditions are used on the upper boundary (figure 2).

The density distribution, shown in figure 2, allows to determine "active" elements of this flow. It contains the contact discontinuity - the jet boundary, and three intersection points. These points are situated on the shock, which brakes the jet. Two of them correspond to arrows in figure 2, and third corresponds to the shock break, which is closer to the symmetry axis (this point disappears and appears again from time to time).

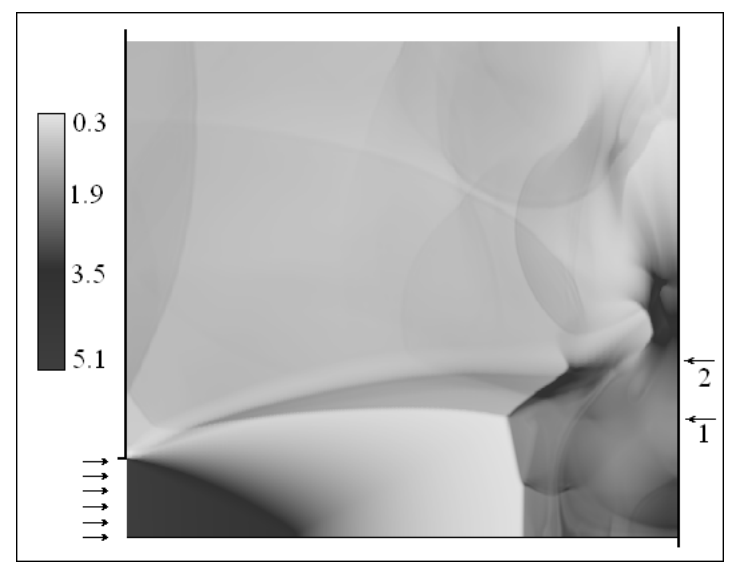

Figure 2. A supersonic jet impinging on a plane, the density distribution

It may be shown [15] that effect of the perturbations reflection from the plane doubles the number of these elements. Thus, there is an ensemble of "active" elements and large number of possible interactions between these elements. It explains existence of unsteady regimes of this flow, according to the proposed mechanism of self-oscillations.

The flow shown in figure 2 is computed with flow conditions $M_{j e t}=2.098$ (Mach number at the exit cross section of the nozzle), $p_{j e t} / p_{\infty}=4.785, \gamma=1.4$ (the specific heat ratio), $h=6.95 r_{j e t}$ ( $h$ - the nozzle exit distance to the right surface, $r_{j e t}$ - the nozzle radius), $\sigma_{j e t}=4^{\circ}$ (the nozzle half-angle). The $696 \times 463$ mesh is used. The resulting frequency of $8206 \mathrm{~Hz}$ may be compared with the experimental frequency of $9033 \mathrm{~Hz}$ [21].

According to the discussed self-oscillations mechanism, the self-oscillations frequency is defined by the distance between interacting "active" elements and the disturbances propagation speed. If to suppose that the dimensionless temperature besides the shock is approximately equal to the jet brake temperature $t=1+M_{j e t}^{2}(\gamma-1) / 2=1+2.098 \mathrm{I} \times 0.2$, consequently, the sound speed is equal to $c=\sqrt{\gamma \times t}=1.62$. The dimensionless self-oscillations period $T=2.24$ corresponds to the frequency of $8206 \mathrm{~Hz}$. So, the distance between "active" elements is equal approximately to 
$L=c \times T / 2=1.82$. This value corresponds to the distance from the brake shock to the right plane (it should be noted that the jet exit radius is taken as a unit, see figure 2). Consequently, an interaction of some intersection point with the plane is responsible for most intensive self- oscillations. To determine which intersection point provides these self-oscillations, time averaged $\overline{S P L}$ s for different places on the plane are computed. Time averaged $\overline{S P L}$ of $203.5 \mathrm{Db}$ is resulted for the $\mathrm{y}=0$ point on the right plane, $\overline{S P L}$ of $197.8 \mathrm{Db}$ for the $\mathrm{y}=1.3$ point (arrow 1), and $\overline{S P L}$ of $208.6 \mathrm{Db}$ for the $\mathrm{y}=2.2$ point (arrow2). Since the oscillations amplitude for the $\mathrm{y}=2.2$ point is about 3 times the amplitude for the $\mathrm{y}=1.3$ point, it seems that the interaction of the intersection point, corresponding to arrow 2 , with the right plane is most intensive in producing of self-oscillations.

\section{Self-Oscillatory Flows near Blunted Bodies, Giving Off Opposite Jets}

Investigations of unsteady interactions of uniform supersonic streams with blunted cones, giving of opposite supersonic jets, were began in $[14,16]$ and are continued here. Calculated flow fields allow to see shocks, contact discontinuities and intersection points. So, these flows may be waited to produce self-oscillations, according to the proposed mechanism of the flow unsteadiness.

Figure 3 shows density levels for the flow with Mach number $M_{\infty}=1.3$ near the spherically blunted cylinder. The conical supersonic jet is running out from a point source located at the sphere centre. The jet half-angle is $\sigma_{j e t}=\arcsin (2 / 3)$, jet Mach number on the surface $x^{2}+y^{2}=r^{2}$ is $M_{j e t}^{j e t}=4.5$, jet pressure is $p_{j e t}=0.23254 p_{\infty}$, jet density is $\rho_{j e t}=0.46455 \rho_{\infty}$. The tangential velocity component is equal to zero on solid surface, other variables are extrapolated. Radial velocity component is equal to zero on the symmetry axis, other variables are extrapolated. Extrapolation conditions are used on the outflow plane, all variables are prescribed on the inflow boundary (figure 3 ).

There are two shocks, namely, the shock on the left side of figure 3, which brakes an outer stream, and the second shock closer to the spherical blunt, which brakes a jet, the extended contact discontinuity between them (denoted by the arrow 1), and the second contact discontinuity, corresponding to the jet boundary. The intersection point (denoted by the arrow 2) of this discontinuity with the shock acts as an amplifier of perturbations. It is important to note that the new contact discontinuity starts from this point and propagates to the region at right side of figure 3 . The contact discontinuity, signed by the arrow 1 , also propagates to this region.

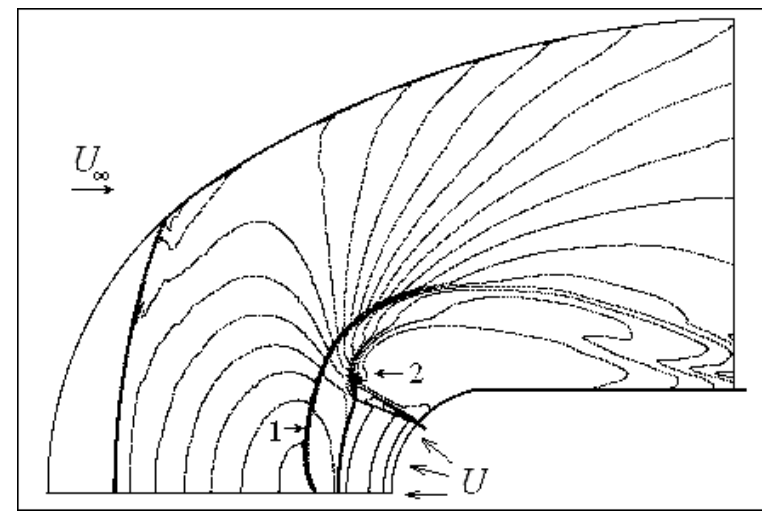

Figure 3. Density levels near the blunted cylinder.

So, if some closed flow contains the tail shock, this shock crosses both contact discontinuities, consequently, two new intersection points appear. This new intersection points may interact one with another or with the body surface and, consequently, may generate self-oscillations. For example, figure 4a shows the density distribution for the flow, defined by presented above parameters, except of the jet angle, which becomes $\sigma_{j e t}=\arcsin (1 / 3)$. This flow contains the tail shock, which starts approximately from the interface point of spherical and cylindrical parts of the body. So, appearing of two additional intersection points on the tail shock is continued by a generation of intensive self-oscillations, producing shocks, moving from the blunt region to the outer shock, where all these shocks become one.

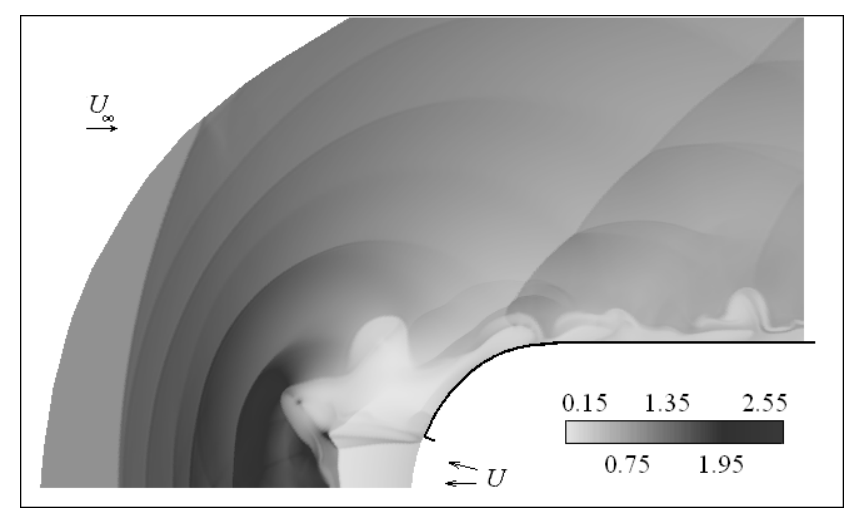

Figure 4a. The density distribution near the blunted cylinder

Figure $4 \mathrm{~b}$ shows the density history at the interface point of spherical and cylindrical parts of the body. Calculations are carried out by third and first order schemes with the identical space stencil. We see that self-oscillations are intensive and do not disappear when implicit highly stable first order scheme is used. Since these intensive self-oscillations appear in the flow, containing the tail shock, the opinion was formulated in [16] about decisive role of this shock in generation of global self-oscillations. 


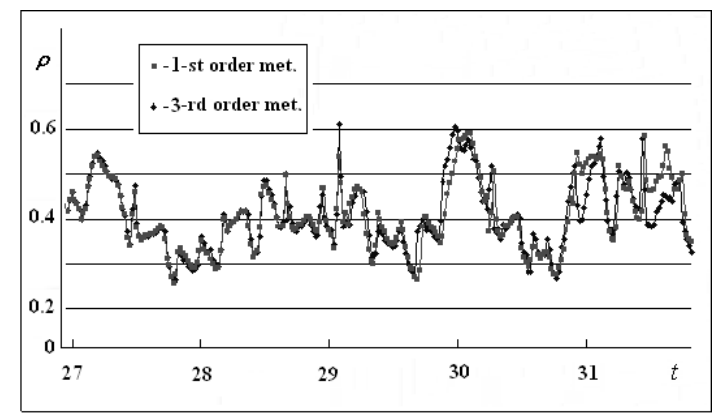

Figure 4b. Density histories at the interface point.

But next investigations shows that situation is more complicated. Now we think, that when the jet angle is diminished from $\sigma_{j e t}=\arcsin (2 / 3)$ to $\sigma_{j e t}=\arcsin (1 / 3)$, the distance from the contact discontinuity, signed by the arrow 1 (see figure 3 ), to the intersection point, signed by the arrow 2 , becomes less, these "active" elements begin to interact more intensively and generation of self-oscillations increases.

To prove this opinion we found a flow which contains the tail shock, but self-oscillations are located near the cylindrical part of the body surface. The flow is calculated with conditions $M_{\infty}=1.3, M_{j e t}=2, p_{j e t}=0.94231 p_{\infty}$, $\rho_{j e t}=0.23771 \rho_{\infty}, \sigma_{j e t}=\arcsin (1 / 3)$. The density distribution (see Figure 5a) shows chaotic waves on the contact discontinuity below the tail shock, but the shocks generation in the region near the body bold is absent. Amplitudes of pressure and density self-oscillations at the interface point of spherical and cylindrical parts of the body are about $15 \%$ of time averaged pressure and density.

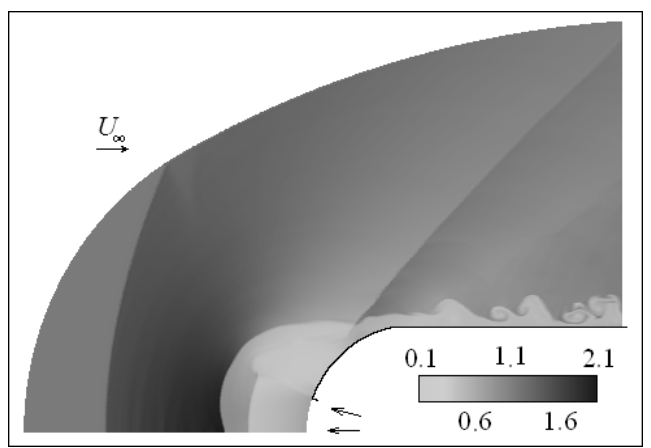

Figure 5a. The density distribution, usage of the approach written in chapter 2.

This flow is calculated from the same initial flow fields with usage of three approaches. Apart of the written in the chapter 2 approach (these results are shown in figure $5 \mathrm{a}$ ), the zero turbulent viscosity (figure $5 b$ ) and the turbulent viscosity calculated by formulas (1) and the constant length scale formulae $z=d$ (figure 5c) are used. Case b corresponds to the Euler equations, case $\mathbf{c}$ is similar to the Smagorinsky approach. It can be seen that the used here approach allows to get the resolution, comparable with the resolution of the Euler equations. It should be noted that a contact discontinuity is very sensitive to a viscosity as a result from the Kelvin-Helmholtz instability. Recent approach deals with the turbulent viscosity, which is independent of the mesh nodes number and allows to check the solutions convergence in this number.

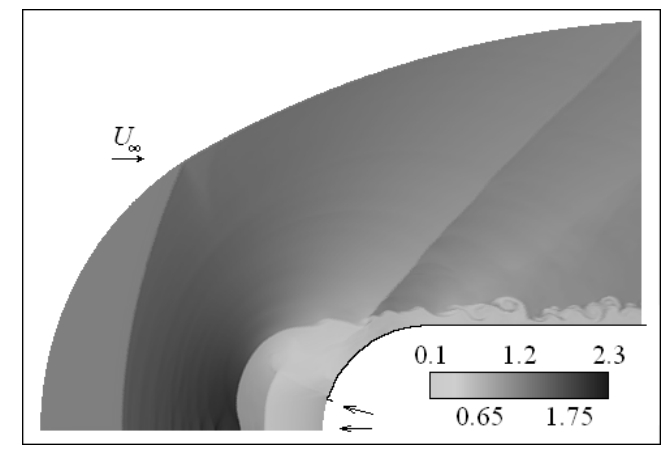

Figure 5b. The density distribution, usage of the.Euler solver

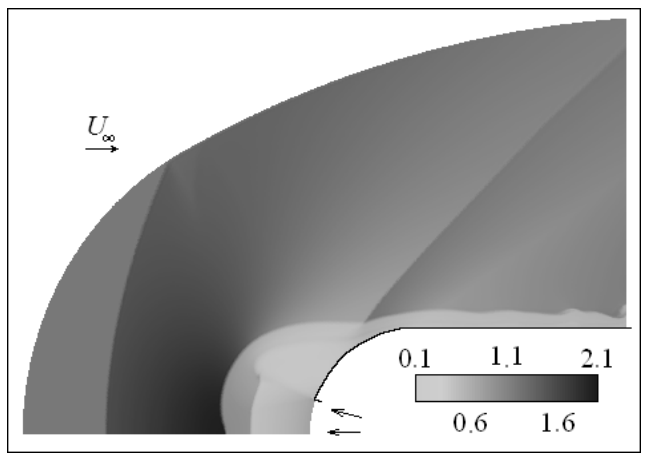

Figure 5c. The density distribution, usage of the constant turbulence length scale.

For example, two meshes $(870 \times 970$ and $653 \times 725)$ are used for computations of the next flow near the blunted cone. Undisturbed stream Mach number is $M_{\infty}=1.3$, the jet half-angle is $\sigma_{j e t}=\arcsin (1 / 3)$, the cone half-angle is $\sigma_{\text {con }}=22.5^{\circ}$, jet Mach number is $M_{\text {jet }}=4.5$, jet pressure is $p_{\text {jet }}=0.23254 p_{\infty}$, jet density is $\rho_{\text {jet }}=0.46455 \rho_{\infty}$. Figure 6a shows the density distribution for the $870 \times 970$ mesh. Intensive self-oscillations are generated in the body bold region. This oscillations produce shocks, propagating to the outer shock.

Figure $6 \mathrm{~b}$ shows pressure histories at the interface point of spherical and conical parts of the body for both meshes. Satisfactory agreement of solutions may be seen.

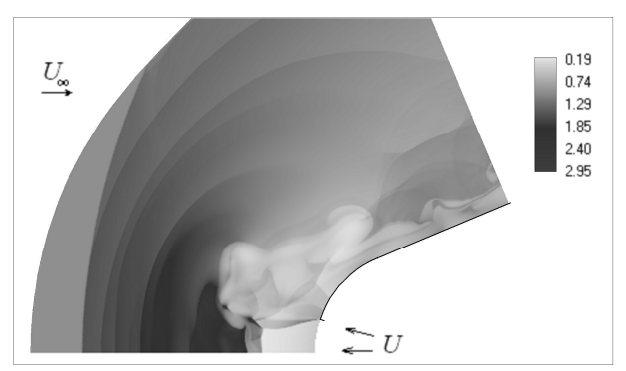

Figure 6a. The density distribution near the blunted cone 


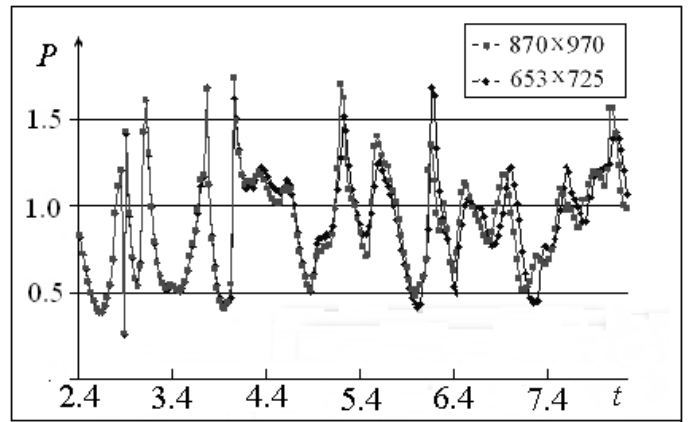

Figure 6b. Pressure histories for two meshes

\section{Conclusions}

Published in previous articles of the author and discussed here the self-oscillations mechanism is based on the main new supposition that intersection points of shocks with shocks or shocks with contact discontinuities may amplify disturbances. These intersection points together with contact discontinuities are "active" elements of any flow, and interactions between these elements provide existence of unsteady regimes. According to this mechanism we tried to determine the main interaction by approximate calculation of the distance between corresponding "active" elements, if the main frequency of oscillations is known (chapter 3 , a jet impinging on a plane). Some new unsteady flows are described here, namely, flows near blunted bodies, giving off supersonic opposite jets. These flows contain two zones, where closed "active" elements are placed. There are different self-oscillatory regimes, due to resonance interactions in the body bold zone or in the tail shock zone or in both zones.

\section{References}

[1] Raman G., Envia E., Bencic T.J., Jet Cavity Interaction Tones, American Institute of Aeronautics and Astronautics J., Vol. 40 (8), 2002, 1503-1511.

[2] Sarpotdar S., Raman G., Cain A.B., Powered Resonance Tubes: Resonance Characteristics and Actuation Signal Directivity, Experiments in Fluids Vol. 39 (6), 2005, 1084-1095.

[3] Raman G., Khanafseh S., Cain A.B., Kerschen E., Development of High Band Width Powered Resonance Tube Actuators with Feedback Control, J. of Sound and Vibration 269 (3-5), 2004, 1031-1062.

[4] Murugappan S., Gutmark E., Parametric Study of the Hartmann-Sprenger Tube, Experiments in Fluids, Vol. 38 (6), 2005, 813-823.

[5] Kastner J., Samimy M., Development and Characterization of Hartmann Tube Fluid Actuators for High-speed Control, American Institute of Aeronautics and Astronautics J., Vol. 40 (10), 2002, 1926-1934.
[6] Henderson B., Bridges J. Wernet, M. An Experimental Study of the Oscillatory Flow Structure of Tone-Producing Supersonic Impinging Jets, J. Fluid Mech., Vol. 542, 2005, 115-137.

[7] Kuo C.-Y., Dowling A. P. Oscillations of a Moderately Underexpanded Choked Jet Impinging Upon a Flat Plate, J. Fluid Mech., Vol. 315, 1996, 267-291.

[8] Berland J., Bogey C., Bailly C. Numerical Study of Screech Generation in a Planar Supersonic Jet, Phys. Fluids, Vol. 19, 2007, 75-105.

[9] Sakakibara Y., Iwamoto J. Numerical Study of Oscillation Mechanism in Underexpanded Jet Impinging on Plate, J. Fluids Eng. , Vol. 120, 1998, 477.

[10] Bodony D. J., Lele S. K. On Using Large-Eddy Simulation for the Prediction of Noise from Cold and Heated Turbulent Jets, Phys. Fluids, Vol. 17, 2005.

[11] Bogey C., Bailly C. Computation of a High Reynolds Number Jet and its Radiated Noise Using Large Eddy Simulation Based on Explicit Filtering, Comput. Fluids, Vol. 35, 2006, 1344-1358.

[12] Cheng T., Lee K. Numerical Simulations of Underexpanded Supersonic Jet and Free Shear Layer Using WENO Schemes, Int. J. Heat Fluid Flow, Vol. 26(5), 2005, 755-770.

[13] Pinchukov V. I., Numerical Modeling of Non-Stationary Flows with Transient Regimes, Comput. Mathem. and Mathem.Phisics, Vol. 49 (10), 2009, 1844-1852.

[14] Pinchukov V. I., Modeling of Self-Oscillations and a Search for New Self-Oscillatory Flows, Mathematical Models and Computer Simulations, Vol. 4(2), 2012, 170-178.

[15] Pinchukov V. I., Self-oscillatory Interactions of Streams, Containing Jets of the Same Direction, with Blunted Bodies Am. J. of Fluid Dynamics, Vol. 3(3), 2013, 80-86.

[16] Pinchukov V. I Modeling of Unsteady Flow near Blunted Cones for Large Time Intervals. Vych. Tekhnol., Vol. 18(1), 2013, 74-86.

[17] Pinchukov V. I., Numerical Solution of the Equations of Viscous Gas by an Implicit Third Order RungeKutta Scheme, Comput. Mathem. and Mathem. Phisics, Vol. 42(6), 2002, 898-907.

[18] Tam C.-J., Orkwis P.D., Disimile P.J. Algebraic Turbulence Model Simulations of Supersonic Open-Cavity Flow Physics, AIAA J., Vol. 34(11), 1996, 2255-2260.

[19] Tam C.-J., Orkwis P.D., Disimile P.J. Comparison of Baldwin-Lomax Turbulence Models for Two-Dimensional Open-Cavity Calculations, AIAA J., Vol. 34(3), Technical Notes , 1996, 629- 632.

[20] Adrianov A. L., Bezrukov A. A., Gaponenko Yu. A., Numerical Study of Interaction of a Supersonic Gas Jet with a Flat Obstacle. Prikl. Mekh. Tekh. Fiz., 41(4), 2000, 106-111.

[21] Gorshkov G. F., Uskov V. N.,. Specialities of SelfOscillations, Arising from Interaction of Supersonic Underexpanded Jet with Finite Obstacle, Prikl. Mekh. Tekh. Fiz., 40(4), 1999, 143-149. 